\title{
Environmental risk from Lyme disease in central and eastern Canada: a summary of recent surveillance information
}

\author{
Ogden $\mathrm{NH}^{1^{*}}$, Koffi $\mathrm{JK}^{1}$, Pelcat $\mathrm{Y}^{2}$, Lindsay $\mathrm{LR}^{2}$ \\ ${ }^{1}$ Centre for Food-borne, Environmental and Zoonotic Infectious Diseases, Public Health Agency of Canada, \\ Saint-Hyacinthe, Quebec \\ ${ }^{2}$ National Microbiology Laboratory, Public Health Agency of Canada, Winnipeg, Manitoba \\ * Corresponding author: nicholas.ogden@phac-aspc.gc.ca
}

\section{Abstract}

Background: Lyme disease is the most commonly reported vector-borne disease in the temperate world. It is emerging in central and eastern Canada due to spread of the tick vector Ixodes scapularis into and within Canada to form new areas of environmental risk known as Lyme disease-endemic areas. Identifying the geographic location of Lyme disease-endemic areas is important to identify the population at risk, target interventions, and inform the clinical diagnosis of Lyme disease patients.

Objective: To provide an up-to-date picture of current and emerging areas of Lyme disease risk in eastern and central Canada by summarizing recent information on Lyme disease-endemic areas, and surveillance for $I$. scapularis ticks.

Methods: Data on locations where I. scapularis have been found in field surveillance studies by a range of federal and provincial organizations were collated and mapped to obtain a fuller picture of the occurrence of $I$. scapularis in Canada. The geographic locations of ticks submitted in passive tick surveillance were mapped for comparison.

Results: The number of confirmed Lyme disease-endemic areas in southern Manitoba, southern and eastern Ontario, southern Quebec, southern New Brunswick and in some locations in Nova Scotia increased from 10 in 2009 to 22 confirmed endemic areas in 2012. The collated field surveillance data indicated that $I$. scapularis tick populations and Lyme disease risk are more geographically widespread than known Lyme disease-endemic areas and that the pattern of emergence of tick populations varies among provinces. There was a tenfold increase in the numbers of $I$. scapularis reported for passive surveillance from 2059 submissions from 1990 to 2003 to 25 738 submissions from 2004 to 2012.

Conclusions: The increasing numbers of Lyme disease-endemic areas, the much wider distribution of tick populations identified by field surveillance, as well as the marked increase in numbers of ticks identified through passive surveillance suggest that the geographic scope of environmental risk of acquiring Lyme disease is expanding in central and eastern Canada, although here it still remains mostly limited to the southern parts of five provinces.

\section{Introduction}

Lyme disease, caused by the bacterium Borrelia burgdorferi, is the most commonly reported vector-borne disease in the temperate zone, particularly affecting North America $(1,2)$. B. burgdorferi is transmitted by ticks, which feed on wildlife reservoir hosts of $B$. burgdorferi, particularly rodents and birds. Ixodes scapularis, the blacklegged tick, is the main vector in eastern and central North America. Ixodes pacificus, the western blacklegged tick, is the main vector west of the Rocky Mountains. Both tick species are indiscriminate in their choice of host and will feed on humans, and in doing so, may transmit pathogens from wildlife to humans. This study focuses on surveillance data for I. scapularis ticks from central and eastern Canada. Studies to obtain data on I. pacificus occurrence in western Canada are underway and will be presented in future articles. 
Risk of infection from B. burgdorferi and other I. scapularis-borne pathogens (Anaplasma phagocytophilum, Babesia microti, Borrelia miyamotoi, a novel Ehrlichia muris-like bacterium, and Powassan virus (3-5) is increasing in eastern and central Canada as populations of $I$. scapularis are spreading north at an estimated rate of $33-55 \mathrm{~km}$ per year (6).

It is important to identify where populations of the tick are becoming established in Canada, because the geographic occurrence of these ticks defines where people can acquire Lyme disease now or in the near future (7). The annual incidence of reported human Lyme disease cases has increased markedly in Canada over the last few years (Figure 1). In 2004 there were 40 reported cases of Lyme disease in Canada; in 2012 there were 315 reported cases. This may reflect an increase in the reporting of cases, but under-reporting might also be occurring (8).

In this paper, we describe where environmental risk of Lyme disease is currently thought to occur based on data of confirmed and suspect Lyme disease-endemic areas, results of a simplified field surveillance method to detect emerging Lyme disease risk areas, and passive tick surveillance, which involves the submission of ticks found attached to patients of participating veterinary and medical clinics. Detection of Lyme disease-endemic areas is the gold standard method of surveillance for Lyme disease risk in the environment, but because it requires multiple site visits over two years it is not very timely or practical. Drag sampling alone is more practical and timely but is less sensitive and specific (9). Passive tick surveillance is a sensitive method of detecting I. scapularis ticks, but is relatively unspecific in terms of the geographic location of tick populations, because small numbers of ticks are dispersed long distances from tick populations by migratory birds. These dispersed ticks are known as adventitious ticks (10).

Figure 1: The number of cases of Lyme disease reported in Canada from 1994 to 2012*

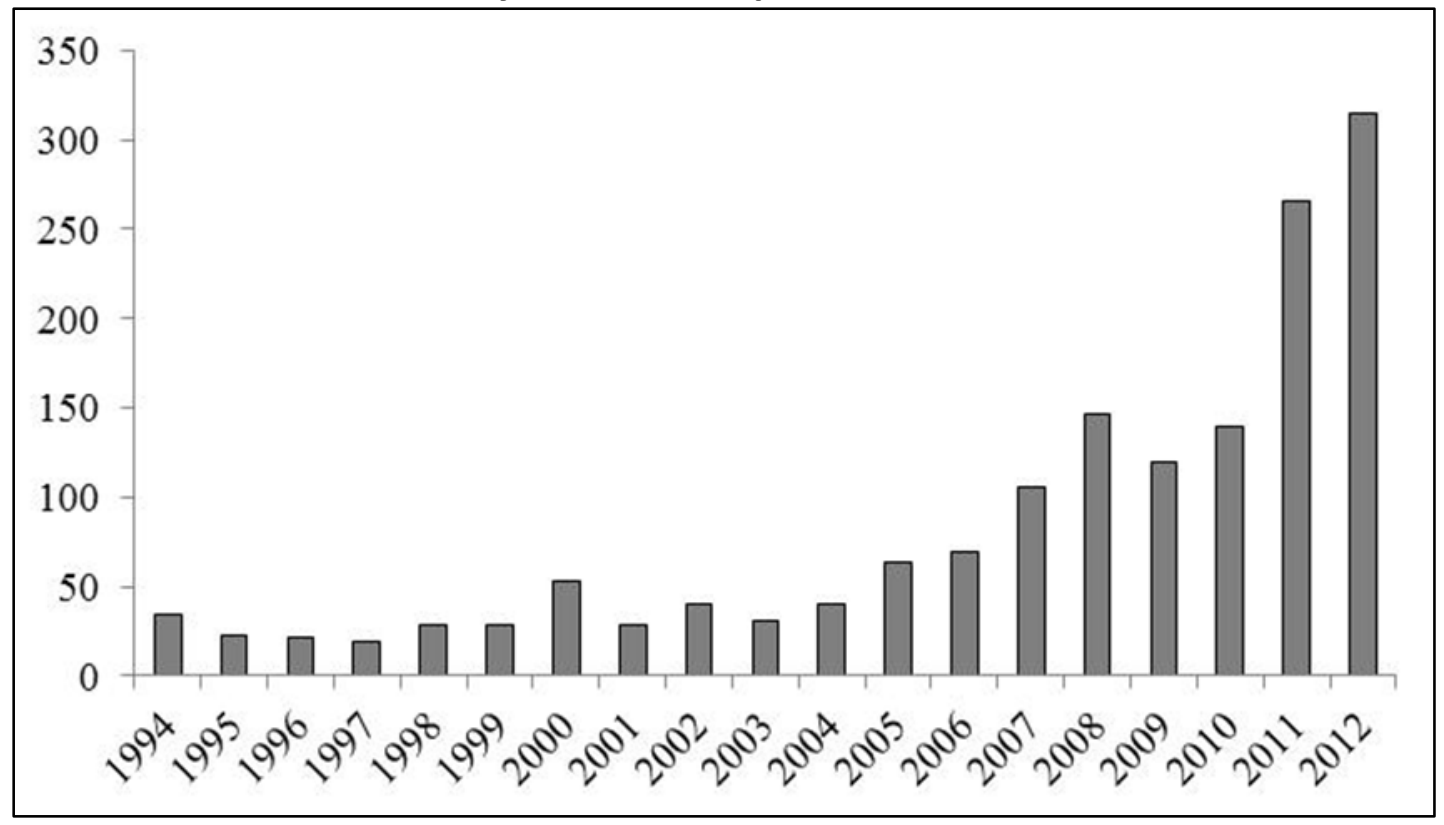

*Numbers of cases before 2009, when Lyme disease became nationally notifiable in Canada, are estimates based on information from provincial public health organizations (10).

Information on where risk is occurring is useful for public health practitioners to identify where to focus prevention and control activities, for health care professionals to assist in diagnosis of Lyme disease, and to assist the public in making informed choices on prevention and control. Information on western Canada is currently pending the results of ongoing field studies. 
The objective of this study was to map out our current knowledge of where the risk of Lyme disease is occurring in central and eastern Canada by:

- Collating knowledge of known Lyme disease-endemic areas;

- Identifying likely emerging Lyme disease-endemic areas detected in field surveillance for ticks;

- Identifying risk posed by adventitious ticks by analysis of passive tick surveillance data.

\section{Methods}

Three types of surveillance data were collated: data on Lyme disease-endemic areas that involved extensive testing, data on field surveillance conducted between 2008 and 2013, and passive tick surveillance collected between 2004 and 2012.

\section{Lyme disease-endemic areas}

Lyme disease-endemic areas are localities where transmission of $B$. burgdorferi by resident populations of vector ticks has been confirmed (11). Confirmation involves drag sampling (trailing a $1-\mathrm{m}^{2}$ square of flannel across the woodland floor for at least three person-hours per site) to collect ticks from the environment that are looking for a blood meal, and the capture of the wild rodents that are important reservoir hosts for $B$. burgdorferi. Detection of all three developmental stages of the tick (larva, nymph, and adult) and B. burgdorferi for more than one year is needed to confirm a location as a Lyme disease-endemic area. Suspect Lyme disease-endemic areas are locations where ticks of more than one developmental stage have been found, where $B$. burgdorferi has been detected in ticks or rodent samples, but where a second year of field sampling has not yet taken place. These known or suspect Lyme disease-endemic areas are based on data provided by provincial public health organizations, and were mapped using ArcGIS Version 10.2 (ESRI).

\section{Field surveillance}

Data were collated on occurrence of $I$. scapularis in field studies conducted by experienced field personnel in collaborations of the Public Health Agency of Canada with provincial government organizations in New Brunswick (2008), Quebec (2010-2012), Manitoba (2010-2012), Nova Scotia (2012), and Ontario (2012-2013). In addition, data from surveillance conducted from 2010 to 2012, and using drag sampling only, were provided by several local public health units in Ontario. In New Brunswick, northwestern Ontario and some sites in Manitoba, both drag sampling (for at least three person-hours per site) and capture of wild rodents (with appropriate ethical approval) were used (12). In all other studies only drag sampling was used.

The species of all ticks in all studies were identified at the Public Health Agency of Canada's National Microbiology Laboratory using standard identification keys. The locations of sampling sites were mapped using ArcGIS Version 10.2 (ESRI). The proportion and exact binomial 95\% confidence intervals of sites on which $I$. scapularis were found in different locations were calculated. As these data were not all gathered contemporaneously or by a standardized technique in terms of season and drag-sampling effort, more detailed statistical analysis was not attempted.

\section{Passive tick surveillance}

The locations of attachment of ticks submitted via provincial public health partners from participating medical and veterinary clinics, and by the general public from 2004 to 2012 were geocoded and mapped in ArcGIS Version 10.2 (ESRI). Only ticks submitted from people or domestic animals that had no history of recent travel were included in the analysis. 


\section{Results}

\section{Lyme disease-endemic areas}

There are currently 18 confirmed and four suspect Lyme disease-endemic areas where I. scapularis has been established; these occur in southern Manitoba, southern and eastern Ontario, southern Quebec, southern New Brunswick and in some locations in Nova Scotia (Figure 2). Some of the Lyme disease-endemic areas in southern Manitoba, southeastern Ontario, and southern Quebec comprise multiple individual locations (15).

Figure 2: The distribution of known (red triangles) and suspect (blue circles) Lyme diseaseendemic areas in Canada

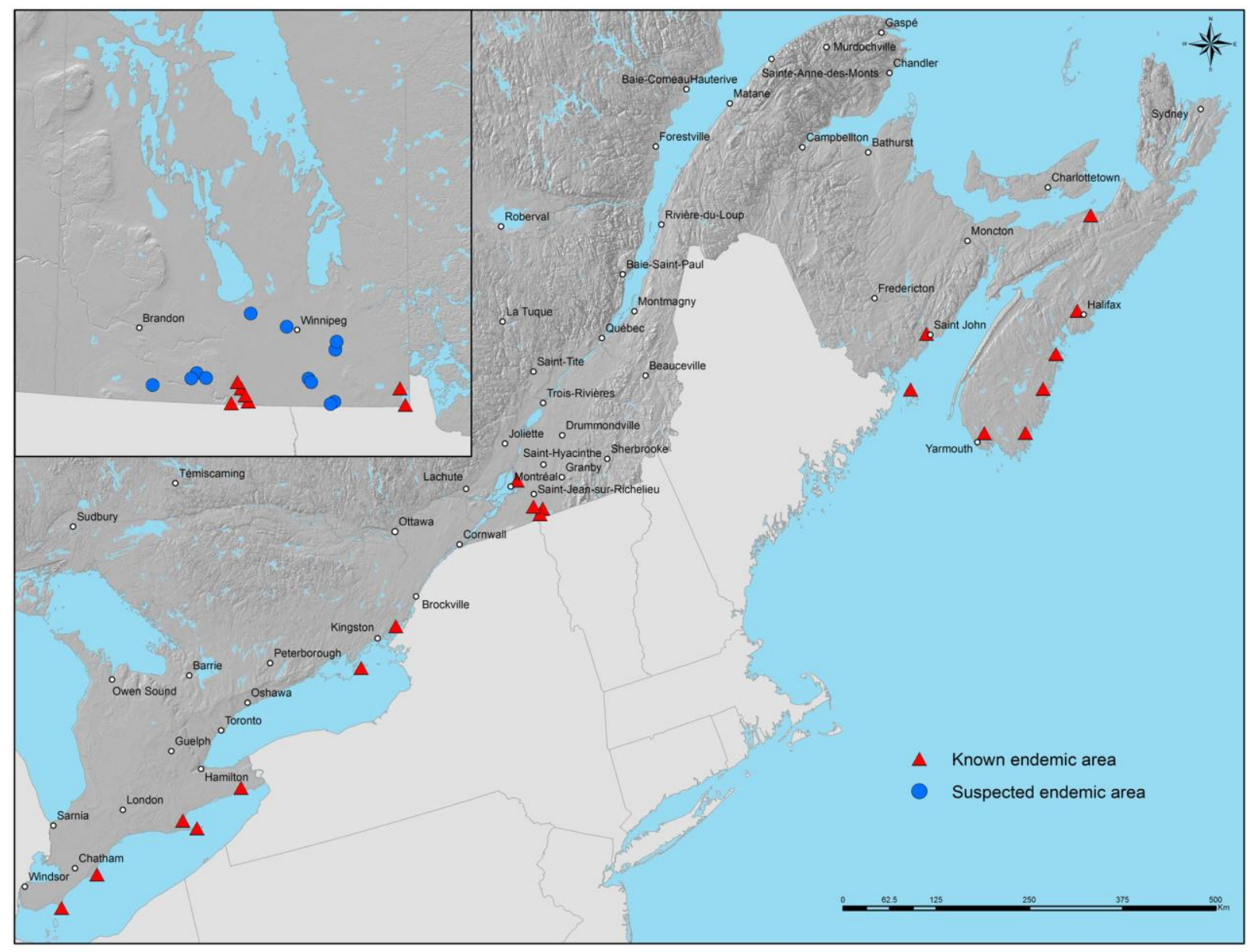




\section{Field surveillance}

Field surveillance data from 296 sites were collated (70 in Manitoba, 87 in Ontario, 73 in Quebec, 16 in New Brunswick, and 50 in Nova Scotia) to identify potentially emerging I. scapularis populations and possible Lyme disease risk (Figure 3).

Figure 3: Results of field surveillance activities for blacklegged ticks in Canada from 2008 to $2012 *$

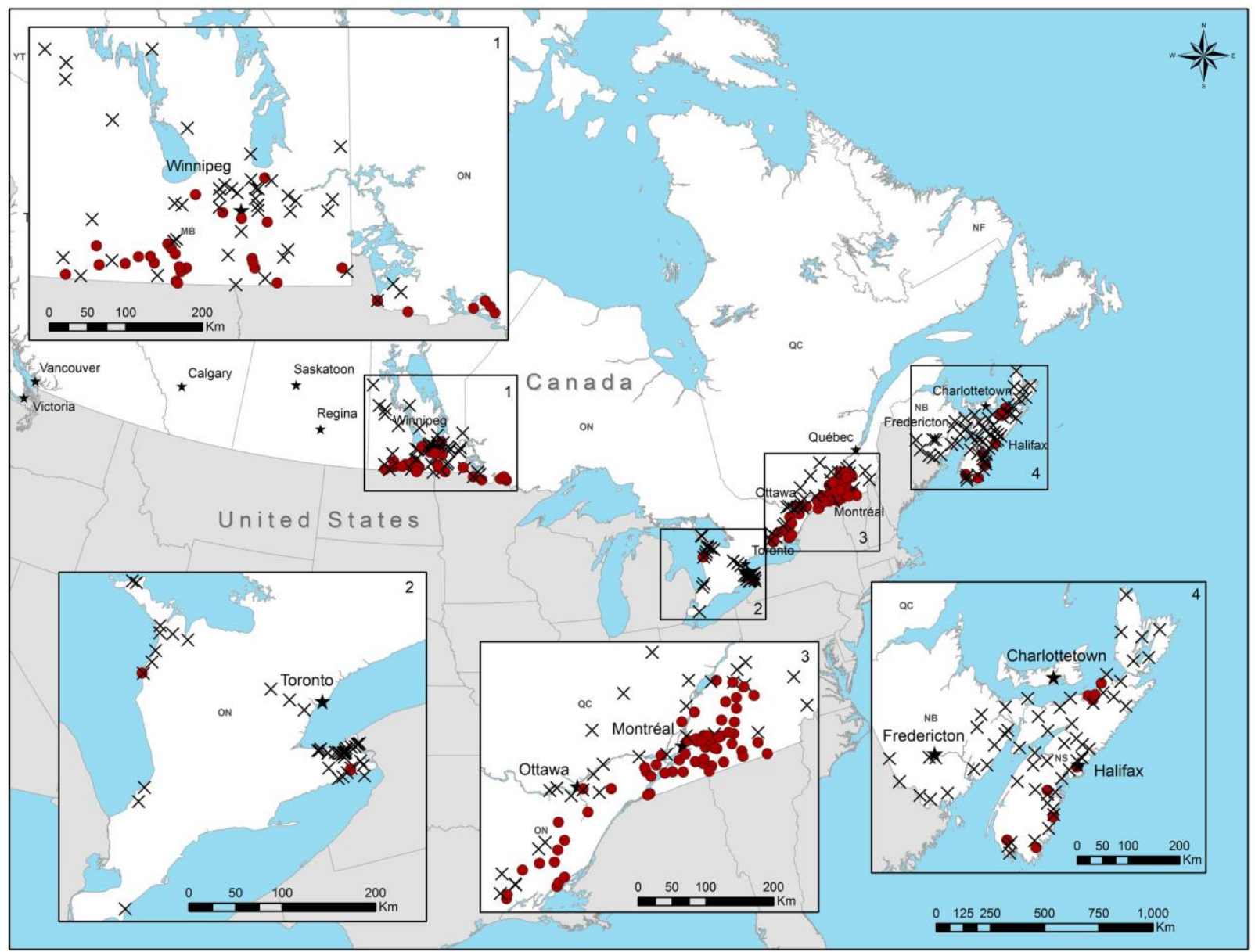

* Sites where at least one I. scapularis tick was found are indicated by filled circles. Sites where I. scapularis were not found are indicated by crosses.

The extent of locations where I. scapularis were found varied from province to province (Figure 3). I. scapularis were found in many woodland sites across southern Quebec and eastern Ontario (effectively comprising a contiguous zone of $I$. scapularis range expansion), and in a region extending across Manitoba south of Winnipeg into northwestern Ontario. Populations of $I$. scapularis are not known to occur at present in Alberta and Saskatchewan. 
We assessed the proportion of sites in a region where I. scapularis ticks were found. In southern Quebec and eastern Ontario, the proportion of $I$. scapularis-positive sites was considerably greater than in the sites in Manitoba north of Winnipeg, in the Golden Horseshoe and Bruce Peninsula regions of Ontario, and in New Brunswick and Nova Scotia (Figure 4).

Figure 4: The proportion of sites visited in field surveillance, in different regions of Canada, at which I. scapularis ticks were found*

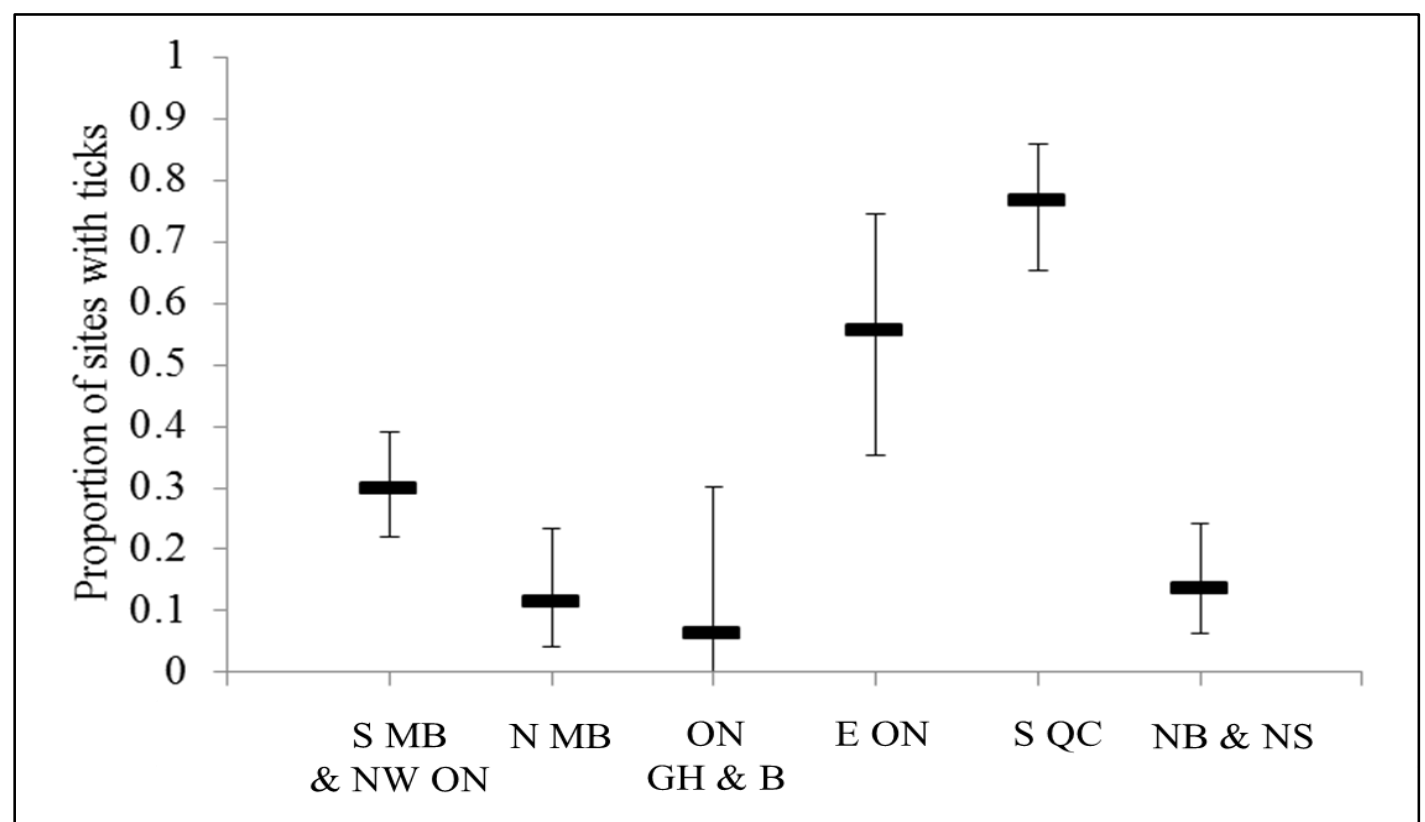

*The regions were Manitoba south of Winnipeg and northwestern Ontario (S MB \& NW ON), Manitoba north of Winnipeg (N MB), Golden Horseshoe and Bruce Peninsula regions of Ontario (ON GH \& B), eastern Ontario (E ON), southern Quebec (S QC), and New Brunswick and Nova Scotia (NB \& NS). The error bars show exact binomial 95\% confidence intervals for the proportion.

\section{Passive tick surveillance}

From 2004 to 2012 there were 25738 individual submissions, comprising 28388 individual ticks, from medical and veterinary clinics in nine Provinces (221 from Alberta, 10 from Saskatchewan, 1063 from Manitoba, 9905 from Ontario, 9371 from Quebec, 1631 from New Brunswick, 829 from Prince Edward Island, 2553 from Nova Scotia, and 155 from Newfoundland). This represents over a tenfold increase compared with the $2059 \mathrm{I}$. scapularis submissions from 1990 to 2003 (10). 
The 2004 to 2012 submissions included 569 that comprised comprising nymphs only, 14 that comprised larvae only, 24925 that comprised adult ticks only, and 230 submissions comprising multiple ticks of more than one tick stage. Interprovincial comparisons of numbers of submitted ticks are not possible due to variations in effort amongst and within provinces, and changes in effort from year to year. The likely locations where ticks were acquired by domestic animals or humans from which they were collected are shown in Figure 5.

Figure 5: The probable locations where I. scapularis ticks submitted from 2004 to 2012 in passive surveillance (acquired via domestic animal and human patients of participating veterinary and medical clinics)

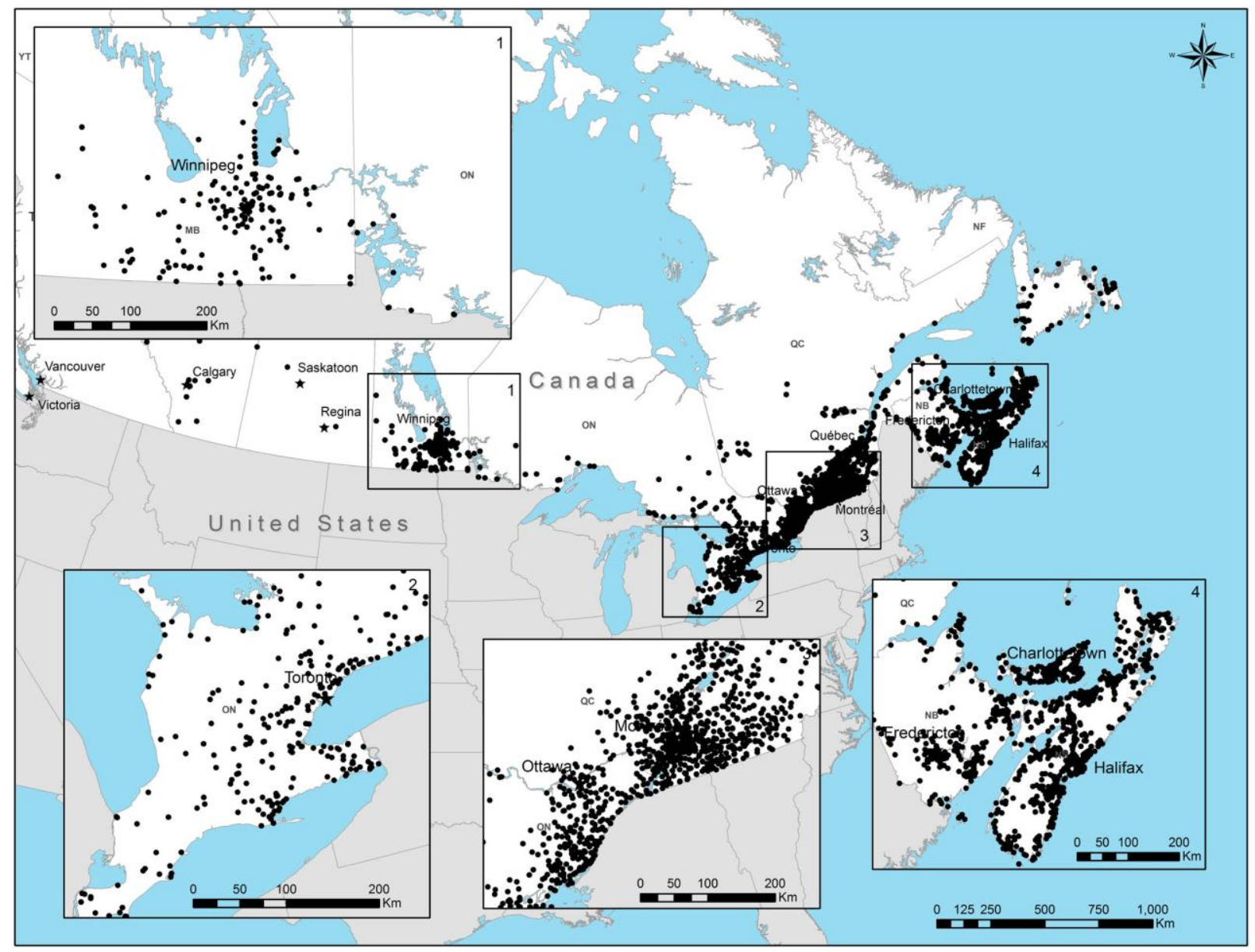

\section{Discussion}

In this study we have presented data on surveillance for the tick vector I. scapularis and for Lyme diseaseendemic areas where the presence of the $B$. burgdorferi has been confirmed. These surveillance data indicate that $I$. scapularis continues to expand its range into southern parts of central and eastern Canada.

Lyme disease-endemic areas are emerging in eastern and central Canada. In 2009 only 10 areas were confirmed in central and eastern Canada (indicated by arrows in Fig 2) (13), whereas now there are now 22 known or suspect Lyme disease-endemic areas in south central and southeastern Canada.

The field surveillance data collated from a number of sources showed that the geographic scope of $I$. scapularis invasion is much greater than that of Lyme disease-endemic areas. The patterns of $I$. scapularis invasion appear to vary from place to place. As expected, invasion is most likely in southern regions of Canada that are closer to 
the United States border, as demonstrated by surveillance in Manitoba. Broad regions of $I$. scapularis invasion are occurring in a contiguous region of southern Manitoba and northwestern Ontario, and in a contiguous region of eastern Ontario and southern Quebec. In southern Ontario west of Toronto, southern New Brunswick, and in Nova Scotia, there was little evidence of Lyme disease risk in the sites visited outside of known Lyme diseaseendemic areas.

Geographic differences in I. scapularis are likely due to differences in rates with which ticks are being carried in from source tick populations, and the factors that determine the suitability of any one location for tick populations to become established (i.e. climate, habitat, and the abundance of suitable wild animal hosts) (14-15). However, in general the field surveillance supports the accuracy of risk maps that have been developed for current and future (with climate change) invasion of I. scapularis (14).

There are a number of limitations to this surveillance study. First, there are no data available from western Canada. We know Lyme disease-endemic areas occur in British Columbia, but Lyme disease risk may be relatively low because I. pacificus is a less efficient vector than I. scapularis (13). There are limitations to the field surveillance data. It is possible that ticks at low densities could have been present on some sites where $I$. scapularis were not found. Results may have also varied due to variations in operator experience, effort per site, and year and month of surveillance. Finally, some of these data date back to 2008 and may no longer reflect the current situation.

The distribution of ticks submitted in passive surveillance was similar to that of tick populations identified in field surveillance. However, this also suggests a low-level risk of exposure of Canadians to Lyme disease in the more northern regions of Manitoba, Ontario, and Quebec, as well as in Newfoundland and Prince Edward Island. This is likely due to ticks being dispersed from established Lyme disease-endemic areas in Canada and the United States by migratory birds and other hosts $(10,16)$. The increasing numbers of Lyme disease-endemic areas, the much wider distribution of tick populations identified by field surveillance, as well as a tenfold increase in the numbers of $I$. scapularis submitted in passive surveillance illustrate the changing landscape of Lyme disease risk in Canada. This is consistent with the increase in reported cases of human Lyme disease.

Future research efforts should be targeted towards integrated surveillance for human cases and $I$. scapularis ticks to identify Lyme disease risk areas and guide the targeting of public health responses. The quantification of the relationship between environmental risk and human case occurrence and incidence is currently under development as part of the national Lyme disease surveillance program. However, expanding surveillance for environmental risk would constitute good public health practice by identifying emerging Lyme disease risk (as well as risk from other $I$. scapularis-borne diseases), and allowing implementation of prevention efforts prior to the occurrence of high numbers of human cases.

\section{References}

(1) Kurtenbach K, Hanincova K, Tsao J, Margos G, Fish D, Ogden NH. Key processes in the evolutionary ecology of Lyme borreliosis. Nat Rev Microbiol. 2006;4:660-69.

(2) Bacon RM, Kugeler KJ, Mead PS, Centers for Disease Control and Prevention (CDC). Surveillance for Lyme disease--United States, 1992-2006. MMWR Surveill Summ. 2008;57(SS10):1-9

(3) Thompson C, Spielman A, Krause PJ. Coinfecting deer-associated zoonoses: Lyme disease, babesiosis, and ehrlichiosis. Clin Infect Dis. 2001;33:676-85.

(4) Pritt BS, Sloan LM, Johnson DK, Munderloh UG, Paskewitz SM, McElroy KM, et al. Emergence of a new pathogenic Ehrlichia species, Wisconsin and Minnesota, 2009. N Engl J Med. 2011;365:422-9.

(5) Krause PJ, Narasimhan S, Wormser GP, Rollend L, Fikrig E, Lepore T, et al. Human Borrelia miyamotoi infection in the United States. N Engl J Med. 2013;368:291-3.

(6) Leighton P, Koffi J, Pelcat Y, Lindsay LR, Ogden NH. Predicting the speed of tick invasion: an empirical model of range expansion for the Lyme disease vector Ixodes scapularis in Canada. J Appl Ecol. 2012;49:457-64. 
(7) Diuk-Wasser MA, Hoen AG, Cislo P, Brinkerhoff R, Hamer SA, Rowland M, et al. Human risk of infection with Borrelia burgdorferi, the Lyme disease agent, in eastern United States. Am J Trop Med Hyg. 2012;86:320-7.

(8) Naleway AL, Belongia EA, Kazmierczak JJ, Greenlee RT, Davis JP. Lyme disease incidence in Wisconsin: a comparison of state-reported rates and rates from a population-based cohort. Am J Epidemiol. 2002;155:1120-7.

(9) Ogden NH, Koffi JK, Lindsay LR. Assessment of a screening test to identify Lyme disease risk. CCDR 2014, Vol 40-5.

(10) Ogden NH, Trudel L, Artsob H, Barker IK, Beauchamp G, Charron D, et al. Ixodes scapularis ticks collected by passive surveillance in Canada: analysis of geographic distribution and infection with the Lyme borreliosis agent Borrelia burgdorferi. J Med Entomol. 2006;43:600-9.

(11) Health Canada. Consensus conference on Lyme disease. CMAJ. 1991;144:1627-32.

(12) Bouchard C, Beauchamp G, Nguon S, Trudel L, Milord F, Lindsay LR, et al. Associations between Ixodes scapularis ticks and small mammal hosts in a newly endemic zone in southeastern Canada: Implications for Borrelia burgdorferi transmission. Ticks Tick Borne Dis. 2011;2:183-90.

(13) Ogden NH, Lindsay LR, Morshed M, Sockett PN, Artsob H. The emergence of Lyme disease in Canada. CMAJ. 2009;180:1221-4

(14) Ogden NH, St-Onge L, Barker IK, Brazeau S, Bigras-Poulin M, Charron DF, et al. Risk maps for range expansion of the Lyme disease vector, Ixodes scapularis, in Canada now and with climate change. Int J Health Geogr. 2008;7:24.

(15) Ogden NH, Mechai S, Margos G. Changing geographic ranges of ticks and tick-borne pathogens: drivers, mechanisms and consequences for pathogen diversity. Front Cell Infect Microbiol. 2013;3:46.

(16) Ogden NH, Bouchard C, Kurtenbach K, Margos G, Lindsay LR, Trudel L, et al. Active and passive surveillance and phylogenetic analysis of Borrelia burgdorferi elucidate the process of Lyme disease risk emergence in Canada. Environ Health Perspect. 2010;118:909-14.

\section{Acknowledgements}

We thank our colleagues in provincial organizations who participated in the field and passive tick surveillance system (Alberta Health, Saskatchewan Health, Manitoba Health, Ontario Agency for Health Promotion and Protection, Institut national de santé publique du Québec and Laboratoire de santé publique du Québec, Université de Montréal, New Brunswick Agriculture, Aquaculture and Fisheries and New Brunswick Health, Nova Scotia Health and Wellness and Department of Natural Resources, Newfoundland \& Labrador Department of Natural Resources), the Hastings \& Prince Edward Counties, Chatham-Kent, Region of Peel, Niagara Region, County of Lambton, Grey Bruce and Northwestern Health Units in Ontario who provided field surveillance data as well as individuals in Canadian universities, veterinarians, and the public who submitted ticks.

\section{Conflict of interest}

There are no conflicts of interest to declare.

\section{Funding}

This work was supported by the Public Health Agency of Canada. 